\title{
ON THE ERSATZ MATERIAL APPROXIMATION IN LEVEL-SET METHODS
}

\author{
Marc Dambrine $^{1}$ And DJalil KATEB $^{2}$
}

\begin{abstract}
The level set method has become widely used in shape optimization where it allows a popular implementation of the steepest descent method. Once coupled with a ersatz material approximation [Allaire et al., J. Comput. Phys. 194 (2004) 363-393], a single mesh is only used leading to very efficient and cheap numerical schemes in optimization of structures. However, it has some limitations and cannot be applied in every situation. This work aims at exploring such a limitation. We estimate the systematic error committed by using the ersatz material approximation and, on a model case, explain that they amplifies instabilities by a second order analysis of the objective function.
\end{abstract}

Mathematics Subject Classification. 49Q10, 34A55, 49Q12.

Received June 26, 2008. Revised March 15, 2009.

Published online July 31, 2009.

\section{INTRODUCTION}

Shape optimization consists in finding the shape of a domain which minimizes an objective function or a criterion. Classical objectives in mechanical engineering are for example, to maximize the torsional rigidity of a body or to minimize its compliance. Such criteria depend on the computation of a state function. In such cases, it usually solves a second-order elliptic partial differential equation that contains the physics under consideration.

Since the pioneering work of Hadamard, a shape calculus has been developed leading to shape derivatives and to subsequent optimization methods. Among the numerous difficulties one has to face during the design of such algorithms, the problem of the eventual change of the number of connected components of the current domain found a satisfactory answer thanks to the level set method introduced by Osher and Sethian [20]. The idea is to define a domain of $\mathbb{R}^{d}$ as the set of points where a real valued function defined on $\mathbb{R}^{d}$ takes its nonpositive values. The geometry can then be discretized as a function on a regular grid and not thanks to control points. The flow of the shape gradient then formally leads to an Hamilton-Jacobi equation for the evolution of the domain coupled with the state function. Recently, Cardaliaguet and Ley have performed in $[7,8]$ a first step in the theoretical justification of this approach on a specific example. However, the level set grid has no particular reason to be useful for the computation of the state function.

In the context of mechanical structure optimization, the boundary value conditions on the part of the structure subject to optimization are usually traction free conditions. In [4], Allaire et al. suggest to take advantage

\footnotetext{
Keywords and phrases. Shape optimization, stability, second order shape derivative, level-set method, ersatz material approximation.

${ }^{1}$ Université de Pau et des Pays de l'Adour; CNRS UMR 5142, LMA, France. marc.dambrine@univ-pau.fr

${ }^{2}$ Université de Technologie de Compiègne; EA 2222, LMAC, France.
} 
of this particular boundary condition to use the level set grid for the numerical computation of the state. The ersatz material approximation consists in filling the outside of the domain by a material of weaker conductivity and replace the state function by the solution of the bimaterial equation stated in the whole level set grid. The physical motivation is that the ersatz material behaves almost like an insulating layer leading to a reasonable approximation of the state that should be computed. This approximation gives very good results for all the usual criteria used in structure optimization and is now a reference method.

However, shape optimization problems are usually unstable. Homogenization is a well known example of unstable shape problem connected to a lack of existence of minimizers. Nevertheless, even in the case where a smooth minimizer exists, the question of stability of such a shape is difficult. In the recent years, the analysis of order two conditions has been studied: A positive second order shape derivative at a critical shape does not imply stability. To sum up, two distinct behaviours of the numerical procedures of optimization have been encountered.

The first example under analysis was the Dirichlet energy. In [13], Descloux showed that the two norms discrepancy phenomenon appears in an electromagnetic shaping problem: the shape hessian at a critical shape is coercive but only in strictly weaker norm than the norm of differentiability. In [10], Dambrine and Pierre have proved that weak information was sufficient to insure stability at the continuous level. This first result was extended in [15] for a numerical scheme.

The second example comes from an inverse problem in electrical impedance tomography reformulated as a question of shape optimization. In $[1,2,14]$, more situations have been studied. The shape hessian at the global minimum is compact and the optimization procedures are severely ill-posed. Hence, appropriate strategies of regularization are required. Since the sequence of eigenvalues of the hessian tend towards zero, there exists a strong link between the number of degrees of freedom used to parameterize the shape and the numerical precision required for the computation of the state. Indeed, the number of shape parameters impose a threshold. Then, if the precision of the computed solution for the state is less than the threshold, the errors committed on the approximated criterion can destroy the well-posedness of the optimization problem.

In this paper, we present a model problem where the parameter of control is the shape of a structure which presents the worst behaviour. We are concerned by a shape optimization problem suggested by the design of micromechanisms introduced in [5] and studied in $[3,11]$. The criterion under consideration has been observed to lead to numerical instabilities (see the comments on the gripping mechanism in [11]). However, we remain for simplicity in the scalar case. The first goal of the paper is to explain rigorously these instabilities by studying the second shape derivative of the criteria in a continuous setting. In particular, in our toy problem, the hessian at the absolute minimizer vanishes. As a consequence of this flatness of the objective to minimize, the ersatz material approximation is prohibited. Indeed, the systematic errors committed on the computation of the state generate instabilities. The second objective of this manuscript is to convince the reader of the interest of second order shape derivatives to understand the behaviour of numerical algorithms of minimization.

The paper is organized as follows. In Section 1, we present the model problem and state the results of this work. Section 2 is devoted to the computation of shape derivatives while the instabilities are discussed in Section 3. The technical results of shape calculus that we need are postponed into an appendix.

\section{Setting of the Problem AND MAin RESUlts}

The shape optimization problem. Let $D$ be a smooth domain of $\mathbb{R}^{d}, d \geq 2$. We suppose that the boundary $\partial D$ of $D$ has three particular disjoint parts $\Gamma_{c}, \Gamma_{d}$ and $\Gamma_{o}$ as illustrated in Figure 1 . Concerning the admissible shapes $\Omega \in \mathcal{U}_{\text {ad }}$, smooth $\mathrm{C}^{2, \alpha}$ subdomain of $D$, we will suppose that

$$
\Gamma_{c} \cup \Gamma_{d} \cup \Gamma_{o} \subset \partial \Omega
$$

Since those parts of $\partial \Omega$ are fixed, the part subject to optimization is the remainder

$$
\Gamma_{m}=\partial \Omega \backslash\left(\overline{\Gamma_{c} \cup \Gamma_{d} \cup \Gamma_{o}}\right) .
$$




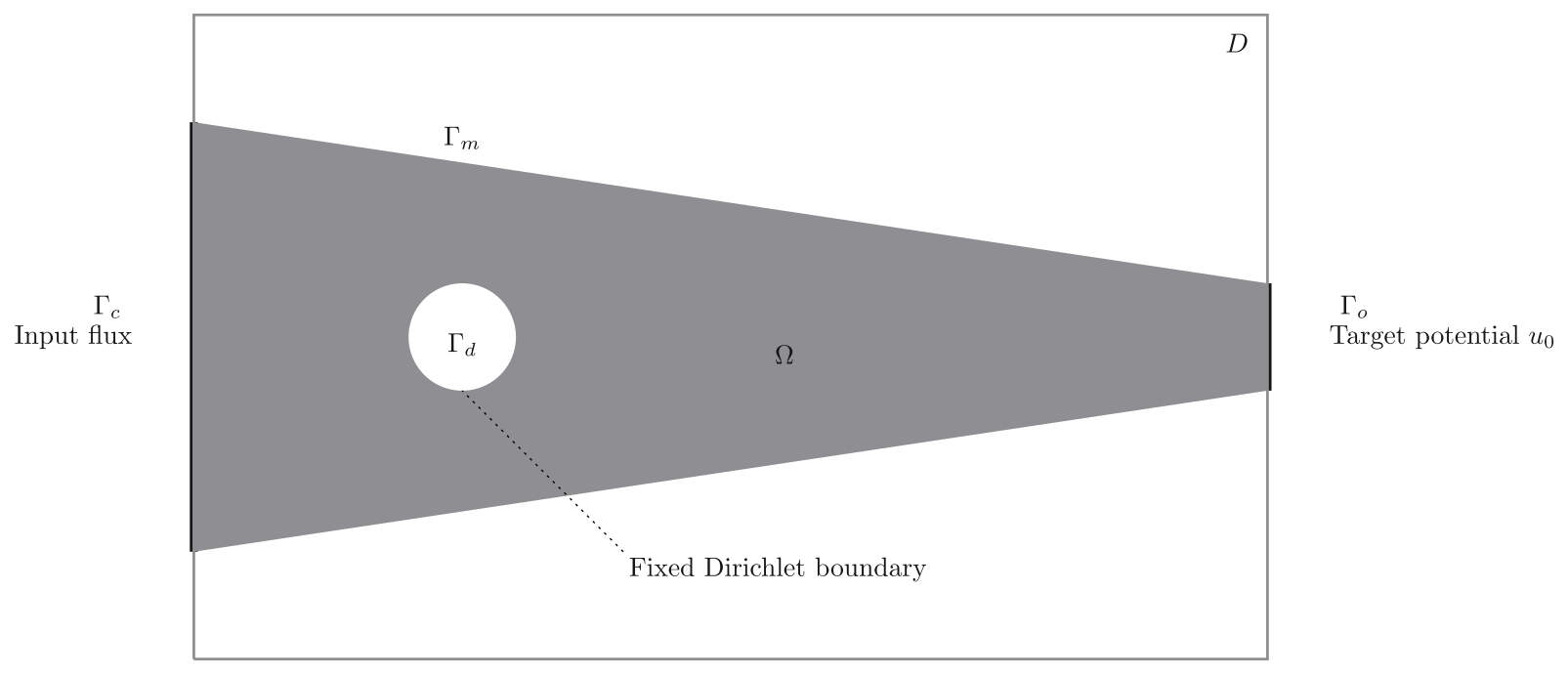

Figure 1. Geometrical settings.

In the sequel, we use the convention that a bold character denotes a vector. For example, $\mathbf{n}$ denotes the normal vector pointing outside of $\Omega$. If $\mathbf{h}$ denotes a deformation field, it can be written as $\mathbf{h}=\mathbf{h}_{\tau}+h_{n} \mathbf{n}$ on $\Gamma_{m}$. Note that $\mathbf{h}_{\tau}$ is a vector while $h_{n}$ is a scalar quantity. The admissible deformation fields have to preserve the complement of $\Gamma_{m}$ in $\partial \Omega$ and therefore the space of admissible fields is

$$
\mathcal{H}=\left\{\mathbf{h} \in \mathrm{C}^{2, \alpha}(D, D), h_{n}=0 \text { on } \Gamma_{c} \cup \Gamma_{d} \cup \Gamma_{o}\right\} .
$$

This problem recovers the following physical meaning: How should one design the structure to find the closest potential distribution to a reference one $u_{0}$ on $\Gamma_{o}$ while imposing the flux on another part of the boundary? More precisely, the state function $u$ solves the following boundary value problem:

$$
\left\{\begin{array}{rll}
-\Delta u=f & \text { in } \Omega, \\
u=0 & \text { on } \Gamma_{d} \\
\partial_{n} u=0 & \text { on } \Gamma_{m} \cup \Gamma_{o}, \\
\partial_{n} u=g & \text { on } \Gamma_{c}
\end{array}\right.
$$

where $f$ and $g$ are $\mathrm{C}^{\infty}$. Both $f$ and $g$ do not live in the natural space but to a stronger one since additional regularity is required in order to define the shape derivatives. Once $\Gamma_{c}$ is of nonnegative measure, this problem admits a unique solution $u \in \mathrm{H}^{1}(\Omega)$.

Let us describe our optimization problem. We consider the criterion $J_{L S}$ defined by

$$
J_{L S}(\Omega)=\frac{1}{2} \int_{\Gamma_{o}}\left|u_{\Omega}-u_{0}\right|^{2} \mathrm{~d} \sigma
$$

where $u_{0} \in H^{\frac{1}{2}}\left(\Gamma_{0}\right)$. The function $u_{\Omega}$ solves (1.1) and hence depends on $\Omega$. For the lightness of notations, we will omit this dependency and denote this function by $u$. We are interested in the minimization of $J_{L S}(\Omega)$ with respect to the shape, where the minimum is taken on the set of admissible domains $\mathcal{U}_{\text {ad }}$. Consequently, the only optimized part of the shape boundary is $\Gamma_{m}$. The Dirichlet boundary conditions are only here to ensure uniqueness of solutions.

For an arbitrary data $u_{0}$, existence of a minimizer to $J_{L S}$ is not clear. Readers interested in this question can refer to Chapter 4 in [16] and the survey article [6]. Nevertheless, this question does not enter the scope 
of this work and we will focus on a well posed problem. We fix an admissible domain $\Omega^{*}$ and choose for data $u_{0}$ exactly the trace on $\Gamma_{o}$ of the solution to the boundary value problem (1.1) set on $\Omega^{*}$. This assumption ensures that the optimization problem has a global minimizer in the admissible domains that is $\Omega^{*}$.

Differentiability results for the state $u$. To compute the shape derivatives, we need some notions of shape optimization postponed in appendix. The following result concerns the first order derivative of the state functions $u$.

Theorem 1.1. Let $\Omega$ be an open smooth subset of $\mathbb{R}^{d}(d \geq 2)$ with a $C^{k, \alpha}$ boundary. Let $\mathbf{h}$ and $\mathbf{h}_{1}, \mathbf{h}_{2}$ be deformation fields in $\mathcal{H}$.

(i) If $k \geq 2$, the state function $u$ is shape differentiable. Its shape derivative $u^{\prime}=D u(\Omega ; \mathbf{h}) \in \mathrm{H}^{1}(\Omega)$ solves the boundary value problem

$$
\left\{\begin{aligned}
\Delta u^{\prime} & =0 \text { in } \Omega \\
u^{\prime} & =0 \text { on } \Gamma_{d} \\
\partial_{n} u^{\prime} & =0 \text { on } \Gamma_{c} \cup \Gamma_{o} \\
\partial_{n} u^{\prime} & =\operatorname{div}_{\tau}\left(h_{n} \nabla_{\tau} u\right)+f h_{n} \text { on } \Gamma_{m} .
\end{aligned}\right.
$$

(ii) If $k \geq 3$, the state $u$ has a second order shape derivative $u^{\prime \prime}=D^{2} u\left(\Omega ; \mathbf{h}_{1}, \mathbf{h}_{2}\right)$ in $\mathrm{H}^{1}(\Omega)$ solution of the boundary value problem

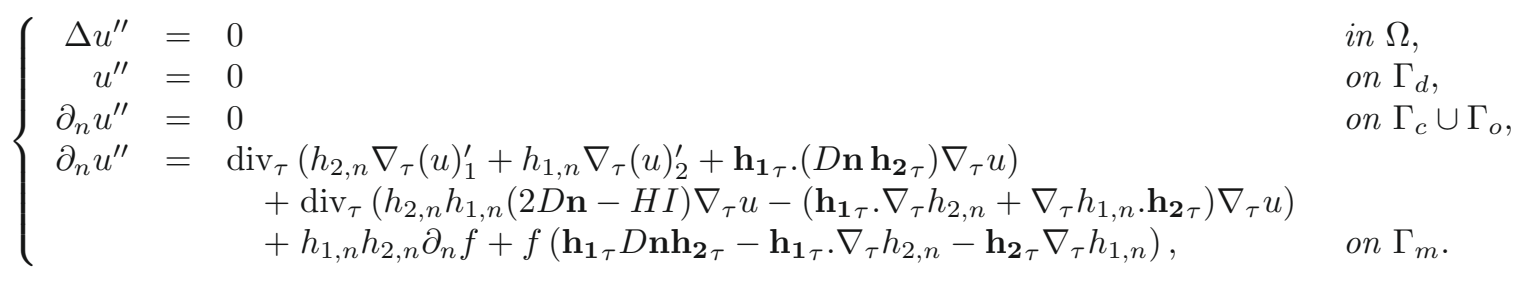

In (1.4), $(u)_{i}^{\prime}$ denotes the first order derivative of $u$ in the direction of $h_{i}$ as given in (1.3), Dn stands for the second fundamental form of the manifold $\Gamma_{m}$ and $H$ stands for its mean curvature.

Differentiability of the objective. Once the differentiability of the state function has been established, the chain rule provides the differentiability with respect to the shape of the criterion. As usual for Least Squares objective, this derivative can be simplified thanks to an adjoint state that will be denoted by $p$.

Proposition 1.2. Let $\Omega$ be an open smooth subset of $\mathbb{R}^{d}(d \geq 2)$ with a $C^{2, \alpha}$ boundary. Then, for all admissible directions $\mathbf{h} \in \mathcal{H}$, the shape derivative of the criteria $D J(\partial \Omega ; \mathbf{h})$ is given by

$$
D J_{L S}(\Omega ; V)=\int_{\Gamma_{m}}\left(f p-\nabla_{\tau} u . \nabla_{\tau} p\right) h_{n}
$$

where the adjoint state $p$ is solution of the adjoint problem

$$
\left\{\begin{aligned}
\Delta p & =0 \text { in } \Omega \\
p & =0 \text { on } \Gamma_{d} \\
\partial_{n} p & =u-u_{0} \text { on } \Gamma_{o} \\
\partial_{n} p & =0 \text { on } \Gamma_{m} \cup \Gamma_{c} .
\end{aligned}\right.
$$

One can also be interested in the computation of higher order derivatives and specially in the second derivative or shape hessian. To that end, we will need the shape derivative of the adjoint state $p$ obtained as a consequence of Theorem 1.1. 
Corollary 1.3. Let $\Omega$ be an open smooth subset of $\mathbb{R}^{d}(d \geq 2)$. Then, the adjoint state function $p$ is shape differentiable. Its shape derivative $p^{\prime}=D p(\Omega ; \mathbf{h}) \in \mathrm{H}^{1}(\Omega)$ solves the boundary value problem

$$
\left\{\begin{aligned}
\Delta p^{\prime} & =0 \text { in } \Omega \\
p^{\prime} & =0 \text { on } \Gamma_{d} \\
\partial_{n} p^{\prime} & =0 \text { on } \Gamma_{c} \cup \Gamma_{o} \\
\partial_{n} p^{\prime} & =\operatorname{div}_{\tau}\left(h_{n} \nabla_{\tau} p\right) \text { on } \Gamma_{m}
\end{aligned}\right.
$$

To investigate the properties of stability of this cost function, we now consider the quadratic form associated to the shape hessian at a critical shape $\Omega_{c}$.

Theorem 1.4. Let $\Omega_{c}$ be an open smooth critical shape for $J_{L S}$ with a $C^{3, \alpha}$ boundary. Let $\mathbf{h}$ be a deformation field in $\mathcal{H}$. The objective $J_{L S}$ is twice differentiable with respect to the shape and its second derivative in the direction $\mathbf{h}$ is given by:

$$
D^{2} J_{L S}\left(\Omega_{c} ; \mathbf{h}, \mathbf{h}\right)=\int_{\Gamma_{m}}\left(f p^{\prime}-\nabla u^{\prime} . \nabla_{\tau} p-\nabla u . \nabla_{\tau} p^{\prime}\right) h_{n}+\partial_{n}\left(f p-\nabla_{\tau} u . \nabla_{\tau} p\right) h_{n}^{2} .
$$

Then, we specify $\Omega_{c}$ : we consider the global minimizer $\Omega^{*}$.

Claim 1.5. If $\Omega^{*}$ realizes the absolute minimum of the criterion $J_{L S}$, then

$$
D^{2} J_{L S}\left(\Omega^{*}, \mathbf{h}, \mathbf{h}\right)=0 .
$$

Equation (1.9) means that the objective is very flat around the minimizer. This fact has two main consequences.

Claim 1.6. On the continuous criterion, the shape hessian at the global minimizer is not coercive. As a consequence, stability of the shape optimization cannot be obtained by classical means (see the criteria of sufficiency of second order conditions in shape optimization [9] and the convergence of numerical scheme obtained in [15] is non insured). However, on this specific example, uniqueness of the minimizer might be obtained by unique continuation arguments. This type of method is of common use in the field of inverse problems [18].

Claim 1.7. The second consequence concerns any numerical scheme used to obtain this optimal domain $\Omega^{*}$. In order to capture the right behaviour of the shape gradient or hessian, one should compute with an extreme precision the state function and its derivative. In the case of a convex objective, the level of the threshold is naturally given by the size of the lowest eigenvalues of the discretized hessian at the critical shape. If the approximation of $u$ inserted in the expression of the shape derivative is computed with a larger error, the computed hessian may have eigenvalues with a non positive real part. In such a case, the numerical descent schemes cannot converge since the discrete objective has no minimum but a saddle point.

The approximation by a ersatz material. One of the main advantage of the level set method in structure optimization is to avoid remeshing of the moving geometry at each step of the evolution. In [4], this is achieved thanks to the ersatz material approximation. In mathematical terms, one approximates the solution $u$ to the boundary value problem (1.1) by the solution $u_{\varepsilon}$ of the new boundary value problem

$$
\left\{\begin{aligned}
-\operatorname{div}\left(\sigma_{\varepsilon} \nabla\right) u_{\varepsilon} & =f & & \text { in } D, \\
u_{\varepsilon} & =0 & & \text { on } \Gamma_{d}, \\
\partial_{n} u_{\varepsilon} & =0 & & \text { on } \partial D \backslash\left(\overline{\Gamma_{c}} \cup \overline{\Gamma_{d}}\right), \\
\partial_{n} u_{\varepsilon} & =g & & \text { on } \Gamma_{c},
\end{aligned}\right.
$$

where $D$ is a domain such that

$$
\Omega \subset D \text { and } \Gamma_{d} \cup \Gamma_{o} \cup \Gamma_{c} \subset \partial D \text {. }
$$


The right hand side $f$ is extended by zero outside of $\Omega$ and the distribution of conductivity $\sigma_{\varepsilon}$ is then such that

$$
\sigma_{\varepsilon}=\chi_{\Omega}+\varepsilon \chi_{D \backslash \bar{\Omega}} .
$$

A very important question is then to know how good is the approximation of $u$ by $u_{\varepsilon}$ ? By variational techniques, Jouve and Murat have shown (personal communication, 2005) that

$$
\left\|u-u_{\varepsilon}\right\|_{\mathrm{H}^{1}(\Omega)} \leq C(\Omega, D, f, g) \sqrt{\varepsilon} .
$$

In fact, numerical simulations show a better order of approximation. We tackle this question with asymptotic methods to recover the order $\varepsilon$.

Theorem 1.8. Let $D$ and $\Omega$ be as in (1.11). Let $u$ (resp. $u_{\varepsilon}$ ) be the solution of the boundary value problem (1.1) (resp. (1.10)). There is a constant $C(\Omega, D, f, g)$ and a value $\varepsilon_{0}>0$ such that

$$
\left\|u-u_{\varepsilon}\right\|_{\mathrm{H}^{1}(\Omega)} \leq C(\Omega, D, f, g) \varepsilon
$$

holds for all $\varepsilon \in\left(0, \varepsilon_{0}\right)$.

The limit of this approach is then clear: in order to keep reasonable matrix to inverse, the value of the parameter $\varepsilon$ cannot be too small: the problem becomes more and more badly conditioned when $\varepsilon$ tends to 0 . This means that the numerical errors committed on the computation of the state function are small but not small enough. With the view to our shape optimization problem with a flat objective, this lack of precision is a serious obstacle to the use of the method. The bad situation depicted in Claim 1.7 appears in the worst case: the hessian provides no possible error.

A better but still highly unstable case is the inverse problem presented in [1] where the hessian with respect of the shape at the global minimizer is non-negative but compact. This phenomenon explains why a parameterized model of the inclusion is used in [1]. It provides the threshold and the mesh used to compute the state function is then appropriately defined.

\section{JustificAtion of the SHAPE DERIVATives}

The section is devoted to the proof of Theorems 1.1 and 1.4. We follow the usual strategy to prove differentiability in shape optimization. Computations made in this section require some classical facts in the context of shape calculus. We present them in Appendix A where we also introduce the notations.

\subsection{Derivatives of the state function. Proof of Theorem 1.1}

Proof of Theorem 1.1.

First order derivatives. Existence of derivatives is well known in that case. Hence we only explain how to derive (1.3). Let $\mathrm{H}_{\Gamma_{d}}^{1}(\Omega)$ the subspace of $\mathrm{H}^{1}(\Omega)$ made of functions that vanishes on $\Gamma_{d}$. We write the weak formulation of (1.1)

$$
\forall \phi \in \mathrm{H}_{\Gamma_{d}}^{1}(\Omega), \quad \int_{\Omega} \nabla u . \nabla \phi+\int_{\Gamma_{c}} g \phi=\int_{\Omega} f \phi .
$$

We then differentiate these integrals with respect to the shape in the direction of $\mathbf{h}$ to obtain

$$
\forall \phi \in \mathrm{H}_{\Gamma_{d}}^{1}(\Omega), \quad \int_{\Omega} \nabla u^{\prime} . \nabla \phi=\int_{\partial \Omega}(f \phi-\nabla u . \nabla \phi) h_{n} .
$$

To be able to interpret the boundary integral as a boundary condition, we need to remove the derivative of $\phi$ in the right hand side. To that end, we use the boundary conditions and the definition of admissible deformation fields

$$
\int_{\partial \Omega} \nabla u . \nabla \phi h_{n}=\int_{\partial \Omega}\left(\nabla_{\tau} u . \nabla_{\tau} \phi+\partial_{n} u \partial_{n} \phi\right) h_{n}=\int_{\partial \Omega} \nabla_{\tau} u . \nabla_{\tau} \phi h_{n} .
$$


Thanks to the integration by part formula (A.7), we get

$$
\int_{\partial \Omega} \nabla_{\tau} u . \nabla_{\tau} \phi h_{n}=-\int_{\Gamma_{m}} \operatorname{div}_{\tau}\left(h_{n} \nabla_{\tau} u\right) \phi
$$

We conclude by density in $\mathrm{L}^{2}\left(\Gamma_{m}\right)$ of the traces of the test functions $\phi$.

Second order derivatives. We compute the second derivative by considering two admissible deformations $\mathbf{h}_{1}, \mathbf{h}_{2} \in \mathcal{H}$ that will describe the small variations of $\partial \Omega$. Simon shows that the second derivative $F^{\prime \prime}\left(\partial \Omega ; \mathbf{h}_{1}, \mathbf{h}_{2}\right)$ of $F(\partial \Omega)$ is defined as a bounded bilinear operator satisfying

$$
F^{\prime \prime}\left(\partial \Omega ; \mathbf{h}_{1}, \mathbf{h}_{2}\right)=\left(F^{\prime}\left(\partial \Omega ; \mathbf{h}_{1}\right)\right)^{\prime} \mathbf{h}_{2}-F^{\prime}\left(\partial \Omega ; D \mathbf{h}_{1} \mathbf{h}_{2}\right) .
$$

For more details, the reader can consult the lecture of Murat and Simon or the book of Henrot and Pierre [16]. We split the proof into two distinct parts: in a first time, we prove existence of the order two derivative, then, in a second time, we show that this derivative solves the boundary value problem (1.4).

First step. Existence of the second derivative

Let us begin the proof. Let $\mathbf{h}_{1}, \mathbf{h}_{2} \in \mathcal{H}$ be two vector fields. The direction $\mathbf{h}_{1}$ being fixed, we consider $\dot{u}_{1, \mathbf{h}_{2}}$ the variation of $\dot{u}_{1}$ with respect to the direction $\mathbf{h}_{2}$. We recall that the material derivative $\dot{u}_{1}$ of $u$ in the direction $\mathbf{h}_{1}$ satisfies

$$
\forall v \in H_{0}^{1}(\Omega), \quad \int_{\Omega} \nabla \dot{u}_{1} \cdot \nabla v=\int_{\Omega} \nabla u \cdot A_{\mathbf{h}_{1}} \nabla v+\int_{\Omega} \operatorname{div}\left(f \mathbf{h}_{1}\right) v .
$$

Let $\phi_{2}: \mathbb{R}^{d} \mapsto \mathbb{R}^{d}$ be the diffeomorphism defined by $\phi_{2}(x)=x+\mathbf{h}_{2}(x)$ and we set $\psi_{2}=\phi_{2}^{-1}$. We introduce the deformed domain $\Omega_{\mathbf{h}_{2}}=\left\{x+\mathbf{h}_{2}(x), x \in \Omega\right\}=\Omega$ to get

$$
\int_{\Omega_{\mathbf{h}_{2}}} \nabla \dot{u}_{1, \mathbf{h}_{2}} \cdot \nabla v=\int_{\Omega_{\mathbf{h}_{2}}} \nabla u_{\mathbf{h}_{2}} \cdot A_{\mathbf{h}_{1}} \nabla v+\int_{\Omega_{\mathbf{h}_{2}}} \operatorname{div}\left(f \mathbf{h}_{1}\right) v
$$

where $u_{\mathbf{h}_{2}}$ is the solution of the original problem with $\Omega_{\mathbf{h}_{2}}$ instead of $\Omega$. Making the change of variables $x=\phi_{2}(X)$, we write the integral identity (2.3) on the fixed domain $\Omega$

$$
\int_{\Omega} \nabla \widetilde{\dot{u}}_{1, \mathbf{h}_{2}} \cdot\left(D \psi_{2}\left(D \psi_{2}\right)^{T} \operatorname{det}\left(D \phi_{2}\right)\right) \nabla v=\int_{\Omega} \nabla \tilde{u}_{\mathbf{h}_{2}} \cdot\left(D \psi_{2} \widetilde{A_{\mathbf{h}_{1}}}\left(D \psi_{2}\right)^{T} \operatorname{det}\left(D \phi_{2}\right)\right) \nabla v+\int_{\Omega} \operatorname{det}\left(D \phi_{2}\right) \widetilde{\operatorname{div}\left(f \mathbf{h}_{1}\right) v}
$$

with the notations $\tilde{u}=u \circ \phi_{2}$ and $\widetilde{A_{\mathbf{h}_{1}}}=A_{\mathbf{h}_{1}} \circ \phi_{2}$. Since the material derivative $\dot{u}_{1}$ of $u$ with respect to the direction $\mathbf{h}_{1}$ satisfies

$$
\int_{\Omega} \nabla \dot{u}_{1} \cdot \nabla v=\int_{\Omega} \nabla u \cdot A_{\mathbf{h}_{1}} \nabla v+\int_{\Omega} \operatorname{div}\left(f \mathbf{h}_{1}\right) v .
$$

The difference of (2.2) and (2.4) gives

$$
\begin{aligned}
\int_{\Omega} \nabla\left(\widetilde{\dot{u}}_{1, \mathbf{h}_{2}}-\dot{u}_{1}\right) \cdot \nabla v= & \int_{\Omega} \nabla \widetilde{\dot{u}}_{1, \mathbf{h}_{2}} \cdot\left(I-D \psi_{2}\left(D \psi_{2}\right)^{T} \operatorname{det}\left(D \phi_{2}\right)\right) \nabla v \\
& +\int_{\Omega} \nabla \tilde{u}_{\mathbf{h}_{2}} \cdot\left(D \psi_{2} \widetilde{A_{\mathbf{h}_{1}}}\left(D \psi_{2}\right)^{T} \operatorname{det}\left(D \phi_{2}\right)-A_{\mathbf{h}_{1}}\right) \nabla v \\
& +\int_{\Omega}\left(\nabla \tilde{u}_{\mathbf{h}_{2}}-\nabla u\right) \cdot A_{\mathbf{h}_{1}} \nabla v+\int_{\Omega}\left(\operatorname{det}\left(D \phi_{2}\right) \widetilde{\operatorname{div}\left(f \mathbf{h}_{1}\right)}-\operatorname{div}\left(f \mathbf{h}_{1}\right)\right) v .
\end{aligned}
$$


We quote from $[17,19]$ the following general asymptotic formulae that contains information on the deformations fields:

$$
\begin{aligned}
& \left\|\operatorname{det}\left(D \phi_{i}\right)-1-\operatorname{div}\left(\mathbf{h}_{i}\right)\right\|_{\infty}=O\left(\left\|\mathbf{h}_{i}\right\|_{\mathrm{C}^{2}}^{2}\right), \\
& \left\|D \psi_{i}\left(D \psi_{i}\right)^{T} \operatorname{det}\left(D \phi_{i}\right)-I+A_{\mathbf{h}_{i}}\right\|_{\infty}=O\left(\left\|\mathbf{h}_{i}\right\|_{\mathrm{C}^{2}}^{2}\right), \\
& \left\|\widetilde{\operatorname{div}\left(h_{1}\right)} \operatorname{det}\left(D \phi_{2}\right)-\operatorname{div}\left(\mathbf{h}_{1}\right)-\operatorname{div}\left(\mathbf{h}_{1}\right) \operatorname{div}\left(\mathbf{h}_{2}\right)-\nabla \operatorname{div}\left(\mathbf{h}_{1}\right) \cdot \mathbf{h}_{2}\right\|_{\infty}=O\left(\left\|\mathbf{h}_{2}\right\|_{\mathrm{C}^{2}}^{2}\right) \text {, } \\
& \| D \psi_{2} \widetilde{A_{\mathbf{h}_{1}}}\left(D \psi_{2}\right)^{T} \operatorname{det}\left(D \phi_{2}\right)-A_{\mathbf{h}_{1}}+D \mathbf{h}_{2} A_{\mathbf{h}_{1}} \\
& +A_{\mathbf{h}_{1}}\left(D \mathbf{h}_{2}\right)^{T}-\operatorname{div}\left(\mathbf{h}_{2}\right) A_{\mathbf{h}_{1}}-\left(A_{\mathbf{h}_{1}}\right)^{\prime}\left(\mathbf{h}_{2}\right) \|_{\infty}=O\left(\left\|\mathbf{h}_{2}\right\|_{\mathrm{C}^{2}}^{2}\right) .
\end{aligned}
$$

Making the adequate substitutions, we easily check that the material derivative of $\dot{u}_{1}$ with respect to $\mathbf{h}_{2}$ exists. This derivative, denoted by $\ddot{u}_{1}$, satisfies

$$
\int_{\Omega} \nabla \ddot{u}_{1} \cdot \nabla v \mathrm{~d} x=\int_{\Omega} \nabla \dot{u}_{1} \cdot A_{\mathbf{h}_{2}} \nabla v+\nabla \dot{u}_{2} \cdot A_{\mathbf{h}_{1}} \nabla v-\nabla u \cdot \mathcal{A} \nabla v+\int_{\Omega} \operatorname{div}\left(\mathbf{h}_{2} \operatorname{div}\left(f \mathbf{h}_{1}\right)\right) v
$$

where $\mathcal{A}$ is defined in (A.12).

Second step. Derivation of (1.4) by formal differentiation of the boundary conditions

The aim of this section is to retrieve the expression of the flux $\partial_{\mathbf{n}} u^{\prime \prime}$ by computing the normal derivatives of each of the expressions $\overline{\nabla u^{\prime} . \mathbf{n}}$ and $\overline{\operatorname{div}_{\tau}\left(h_{1, n} \nabla_{\tau} u\right)}$. Since

$$
\nabla u^{\prime} \cdot \mathbf{n}=\operatorname{div}_{\tau}\left(h_{1, n} \nabla_{\tau} u\right)+f h_{1, n}=h_{1, n} \Delta_{\tau} u+\nabla_{\tau} h_{1, n} \cdot \nabla_{\tau} u+f h_{1, n}
$$

then, after taking the material derivative of the two sides of the above identity we obtain

$$
\begin{aligned}
& \overline{\nabla u^{\prime} \cdot \mathbf{n}}=\overline{\operatorname{div}_{\tau}\left(h_{1, n} \nabla_{\tau} u\right)}+\dot{f h_{1, n}} \\
& =\dot{\overline{h_{1, n}}} \Delta_{\tau} u+h_{1, n} \overline{\dot{\Delta_{\tau} u}}+\overline{\dot{\nabla_{\tau} h_{1, n}}} \cdot \nabla_{\tau} u+\nabla_{\tau} h_{1, n} \cdot \dot{\overline{\nabla_{\tau} u}}+\dot{f} h_{1, n}+f \dot{f} \overline{h_{1, n}} .
\end{aligned}
$$

In order to avoid lengthy computations, we shall concentrate on each normal derivative appearing in the above formula. Some results are straightforward and their proof will be then left to the reader. Thanks to Proposition A.7, we conclude that

$$
\overline{\nabla_{\tau} h_{1, n}}=-\nabla_{\tau}\left(\mathbf{h}_{1} \cdot \nabla_{\tau} h_{2, n}\right)+\left(D^{2} h_{1, n} \cdot \mathbf{h}_{2}\right)_{\tau}-\nabla h_{1, n} \cdot \dot{\mathbf{n}} \mathbf{n}-\nabla h_{1, n} \cdot \mathbf{n} \dot{\mathbf{n}} .
$$

In the same manner, we also get

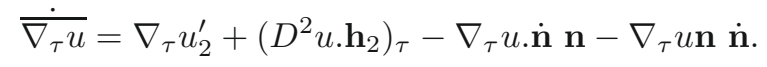

Hence, we can write

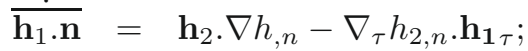

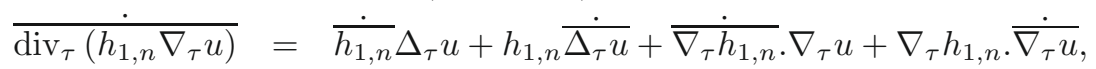

$$
\begin{aligned}
& =\left(-\nabla_{\tau}\left(\mathbf{h}_{1} \cdot \nabla_{\tau} h_{2, n}\right)+\left(D^{2} h_{1, n} \cdot \mathbf{h}_{2}\right)_{\tau}-\nabla h_{1, n} \cdot \mathbf{n} \dot{\mathbf{n}}\right) \nabla_{\tau} u+h_{1, n} \dot{\dot{\Delta_{\tau} u}} \\
& +\nabla_{\tau} h_{1, n}\left(\nabla_{\tau} u_{2}^{\prime}+\left(D^{2} u \cdot \mathbf{h}_{2}\right)_{\tau}\right)+\left(\mathbf{h}_{2} \cdot \nabla h_{1, n}-\nabla_{\tau} h_{2, n} \cdot \mathbf{h}_{\mathbf{1}_{\tau}}\right) \cdot \Delta_{\tau} u .
\end{aligned}
$$

It remains to simplify the terms $A=\left(D^{2} u \cdot \mathbf{h}_{2}\right)_{\tau} \cdot \nabla_{\tau} h_{1, n}$ and $B=\nabla_{\tau} u \cdot\left(D^{2} h_{1, n} \cdot \mathbf{h}_{2}\right)_{\tau}$. We obtain:

$$
\begin{aligned}
& A=-\nabla_{\tau} u \cdot\left(D \mathbf{n} \nabla_{\tau} h_{1, n}\right) h_{2, n}+D^{2} u \mathbf{h}_{\mathbf{2} \tau} \cdot \nabla_{\tau} h_{1, n} \\
& B=\nabla_{\tau}\left(\partial_{\mathbf{n}} h_{1, n}\right) \cdot \nabla_{\tau} u h_{2, n}-\nabla_{\tau} u \cdot\left(D \mathbf{n} \nabla_{\tau} h_{1, n}\right) h_{2, n}+\left(D^{2} h_{1, n} \cdot \mathbf{h}_{\mathbf{2} \tau}\right) \cdot \nabla_{\tau} u .
\end{aligned}
$$


We tackle the computation of $\left(\partial_{\mathbf{n}} u^{\prime}\right)^{\prime}$. We first expand $\overline{\operatorname{div}_{\tau}\left(h_{1, n} \nabla_{\tau} u\right)}$ :

$$
\overline{\operatorname{div}_{\tau}\left(h_{1, n} \nabla_{\tau} u\right)}=\overline{\dot{h_{1, n} \Delta_{\tau} u}}+\overline{\nabla_{\tau} h_{1, n} \cdot \nabla_{\tau} u}=\dot{\overline{h_{1, n}}} \Delta_{\tau} u+h_{1, n} \overline{\dot{\Delta_{\tau} u}}+\overline{\nabla_{\tau} h_{1, n}} \cdot \nabla_{\tau} u+\nabla_{\tau} h_{1, n} \cdot \dot{\overline{\nabla_{\tau} u}} .
$$

After substitution, one gets

$$
\begin{aligned}
& \overline{\operatorname{div}_{\tau}\left(h_{1, n} \nabla_{\tau} u\right)}=\operatorname{div}_{\tau}\left(h_{1, n} \nabla_{\tau} u_{2}^{\prime}\right)+\operatorname{div}\left(\left(h_{2, n} \partial_{\mathbf{n}} h_{1, n}-\nabla_{\tau} h_{2, n} \cdot \mathbf{h}_{\mathbf{1}_{\tau}}\right) \nabla_{\tau} u\right)+\nabla_{\tau} u \cdot\left(D^{2} h_{1, n} \cdot \mathbf{h}_{\mathbf{2} \tau}\right) \\
& -\partial_{\mathbf{n}} h_{1, n} \nabla_{\tau} u .\left(D \mathbf{n} \mathbf{h}_{\mathbf{2} \tau}\right)-2 h_{2, n} \nabla_{\tau} u .\left(D \mathbf{n} \nabla_{\tau} h_{1, n}\right)+D^{2} u \mathbf{h}_{\mathbf{2} \tau} . \nabla_{\tau} h_{1, n} \\
& +\Delta_{\tau} u \nabla_{\tau} h_{1, n} \cdot \mathbf{h}_{\mathbf{2} \tau}+h_{1, n}\left(\dot{\overline{\Delta_{\tau} u}}-\Delta_{\tau} u_{2}^{\prime}\right) \text {. }
\end{aligned}
$$

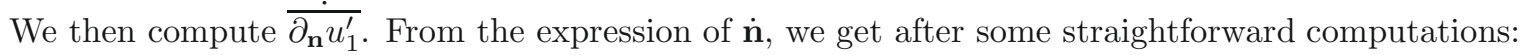

$$
\overline{\partial_{\mathbf{n}} u_{1}^{\prime}}=\partial_{\mathbf{n}}\left(u_{1}^{\prime}\right)_{2}^{\prime}+\left(D^{2} u_{1}^{\prime} \mathbf{h}_{2}\right) \cdot \mathbf{n}+\nabla_{\tau} u_{1}^{\prime} \cdot\left(D \mathbf{n} \mathbf{h}_{\mathbf{2} \tau}-\nabla_{\tau} h_{2, n}\right) .
$$

Finally, we compute $\partial_{\mathbf{n}}\left(u_{1}^{\prime}\right)_{2}^{\prime}$. We deduce from (2.7), (2.8) and formula

$$
\overline{\nabla_{\tau} h_{1, n}}=\overline{\operatorname{div}_{\tau}\left(h_{1, n} \nabla_{\tau} u\right)}+\dot{f} h_{1, n}+f \frac{\dot{h_{1, n}}}{h_{1}}
$$

a first expression of the normal derivative of the second order shape derivative

$$
\begin{aligned}
& \partial_{n}\left(u_{1}^{\prime}\right)_{2}^{\prime}=\overline{\operatorname{div}_{\tau}\left(h_{1, n} \nabla_{\tau} u\right)}-\left(D^{2} u_{1}^{\prime} \mathbf{h}_{2}\right) \cdot \mathbf{n}-\nabla_{\tau} u_{1}^{\prime} \cdot\left(D \mathbf{n} \mathbf{h}_{\mathbf{2}_{\tau}}-\nabla_{\tau} h_{2, n}\right) \\
& =\operatorname{div}_{\tau}\left(h_{1, n} \nabla_{\tau} u_{2}^{\prime}+\left(h_{2, n} \partial_{\mathbf{n}} h_{1, n}-\nabla_{\tau} h_{2, n} \cdot \mathbf{h}_{\mathbf{1}_{\tau}}\right) \nabla_{\tau} u\right)-\partial_{\mathbf{n}} h_{1, n} \nabla_{\tau} u \cdot\left(D_{n} \mathbf{h}_{\mathbf{2} \tau}\right) \\
& +\nabla_{\tau} u_{1}^{\prime} \cdot\left(\nabla_{\tau} h_{2, n}-D \mathbf{n} \mathbf{h}_{2}\right)+\left(D^{2} h_{1, n} \mathbf{h}_{\mathbf{2}_{\tau}}\right) \cdot \nabla_{\tau} u+D^{2} u \mathbf{h}_{\mathbf{2} \tau} \cdot \nabla_{\tau} h_{1, n}+\dot{f} h_{1, n}+f \frac{\dot{f} \overline{h_{1, n}}}{(\bar{h}} \\
& -2 h_{2, n}\left(D \mathbf{n} \nabla_{\tau} u\right) . \nabla_{\tau} h_{1, n}+\nabla_{\tau} h_{1, n} \cdot \mathbf{h}_{2} \Delta_{\tau} u+h_{1, n}\left(\dot{\overline{\Delta_{\tau} u}}-\Delta_{\tau} u_{2}^{\prime}\right)-\left(D^{2} u_{1}^{\prime} \mathbf{h}_{2}\right) \cdot \mathbf{n} .
\end{aligned}
$$

We have

$$
\dot{f} h_{1, n}+f \dot{f} \overline{h_{1, n}}=\nabla f \cdot \mathbf{h}_{2}+f\left(\mathbf{h}_{2} \cdot \nabla h_{1, n}-\nabla_{\tau} h_{2, n} \cdot \mathbf{h}_{\mathbf{1} \tau}\right) .
$$

Taking account of the following calculation,

$$
\begin{aligned}
-\left(D^{2} u_{1}^{\prime} \mathbf{h}_{2}\right) \cdot \mathbf{n}+\nabla_{\tau} u_{1}^{\prime} \cdot \nabla_{\tau} h_{2}, n & =-\left(h_{2, n} D^{2} u_{1}^{\prime} \mathbf{n}+D^{2} u_{1}^{\prime} \mathbf{h}_{2 \tau}\right) \cdot \mathbf{n}+\nabla_{\tau} u_{1}^{\prime} \cdot \nabla_{\tau} h_{2}, n, \\
& =h_{2, n}\left(\Delta_{\tau} u_{1}^{\prime}+H \partial_{\mathbf{n}} u_{1}^{\prime}\right)+\nabla_{\tau} u_{1}^{\prime} \cdot \nabla_{\tau} h_{2, n}-\left(D^{2} u^{\prime} 1 \mathbf{h}_{\mathbf{2} \tau}\right) \cdot \mathbf{n}, \\
& =\operatorname{div}_{\tau}\left(h_{2, n} \nabla_{\tau} u_{1}^{\prime}\right)+H h_{2, n} \partial_{\mathbf{n}} u_{1}^{\prime}-\left(D^{2} u_{1}^{\prime} \mathbf{h}_{2}\right) \cdot \mathbf{n} ;
\end{aligned}
$$

we rewrite $\partial_{\mathbf{n}}\left(u_{1}^{\prime}\right)_{2}^{\prime}$ as

$$
\begin{aligned}
& \partial_{\mathbf{n}}\left(u_{1}^{\prime}\right)_{2}^{\prime}=\operatorname{div}_{\tau}\left(h_{1, n} \nabla_{\tau} u_{2}^{\prime}+h_{2, n} \nabla_{\tau} u_{1}^{\prime}+\left(h_{2, n} \partial_{\mathbf{n}} h_{1, n}-\nabla_{\tau} h_{2, n} \cdot \mathbf{h}_{\mathbf{1}_{\tau}}\right) \nabla_{\tau} u\right) \\
& -\left(\nabla_{\tau} u_{1}^{\prime}+\partial_{\mathbf{n}} h_{1, n} \nabla_{\tau} u\right) \cdot\left(D \mathbf{n} \mathbf{h}_{\mathbf{2}_{\tau}}\right)+H h_{2, n} \partial_{\mathbf{n}} u_{1}^{\prime}-\left(D^{2} u_{1}^{\prime} \mathbf{h}_{\mathbf{2} \tau}\right) \cdot \mathbf{n} \\
& +\left(D^{2} h_{1, n} \mathbf{h}_{\mathbf{2} \tau}\right) \cdot \nabla_{\tau} u+D^{2} u \mathbf{h}_{\mathbf{2} \tau} \cdot \nabla_{\tau} h_{1, n}-2 h_{2, n} \nabla_{\tau} h_{1, n} \cdot\left(D \mathbf{n} \nabla_{\tau} u\right)+\nabla f \cdot \mathbf{h}_{2} \\
& +\Delta_{\tau} u \nabla_{\tau} h_{1, n} \cdot \mathbf{h}_{\mathbf{2} \tau}+h_{1, n}\left(\dot{\overline{\Delta_{\tau} u}}-\Delta_{\tau} u_{2}^{\prime}\right)+f\left(\mathbf{h}_{2} \cdot \nabla h_{1, n}-\nabla_{\tau} h_{2, n} \cdot \mathbf{h}_{\mathbf{1} \tau}\right) \text {. }
\end{aligned}
$$

This formula remains hard to handle. To get a more convenient one, we have to work a bit more. First, we derive tangentially to the direction $\mathbf{h}_{2}$ the boundary identity

$$
\partial_{\mathbf{n}} u_{1}^{\prime}=h_{1, n} \Delta_{\tau} u+\nabla_{\tau} h_{1, n} \cdot \sigma \nabla_{\tau} u+f h_{1, n} .
$$


This leads to:

$$
\begin{aligned}
& \left(D^{2} u_{1}^{\prime} \mathbf{h}_{\mathbf{2}_{\tau}}\right) \cdot \mathbf{n}+\left(D \mathbf{n} \mathbf{h}_{\mathbf{2}_{\tau}}\right) \cdot \nabla_{\tau} u_{1}^{\prime}=\nabla_{\tau} h_{1, n} \cdot \mathbf{h}_{\mathbf{2}_{\tau}} \Delta_{\tau} u+h_{1, n} \nabla_{\tau} \Delta_{\tau} u \cdot \mathbf{h}_{\mathbf{2} \tau} \\
& +\left(D^{2} h_{1, n} \mathbf{h}_{\mathbf{2}_{\tau}}\right) \cdot \nabla_{\tau} u-\partial_{\mathbf{n}} h_{1, n} \nabla_{\tau} u .\left(D \mathbf{n} \mathbf{h}_{\mathbf{2}_{\tau}}\right)+D^{2} u \mathbf{h}_{\mathbf{2} \tau} \cdot \nabla_{\tau} h_{1, n} \\
& +\nabla_{\tau} f \cdot \mathbf{h}_{\mathbf{2} \tau} h_{1, n}+f \nabla_{\tau} h_{1, n} \cdot \mathbf{h}_{\mathbf{2} \tau} \text {. }
\end{aligned}
$$

From (A.11) and subtracting (2.10) from (2.9), we can write

$$
\begin{aligned}
\partial_{\mathbf{n}}\left(u_{1}^{\prime}\right)_{2}^{\prime}= & \operatorname{div}_{\tau}\left(h_{1, n} \nabla_{\tau} u_{2}^{\prime}+h_{2, n} \nabla_{\tau} u_{1}^{\prime}+\left(h_{2, n} \partial_{\mathbf{n}} h_{1, n}-\nabla_{\tau} h_{2, n} \cdot \mathbf{h}_{\mathbf{1} \tau}\right) \nabla_{\tau} u\right) \\
& +\operatorname{div}_{\tau}\left(h_{1, n} h_{2, n}(H I-2 D \mathbf{n}) \cdot \nabla_{\tau} u\right)-h_{1, n}\left(\nabla_{\tau} \Delta_{\tau} u \cdot \mathbf{h}_{\mathbf{2}_{\tau}}+\Delta_{\tau} \nabla_{\tau} u \cdot \mathbf{h}_{\mathbf{2} \tau}\right) \\
& +h_{1, n}\left(\nabla_{\tau} \operatorname{div}_{\tau}\left(\mathbf{h}_{\mathbf{2} \tau}\right) \cdot \nabla_{\tau} u-\operatorname{div}_{\tau}\left(\left(\left(D \mathbf{h}_{2}+\left(D \mathbf{h}_{2}\right)^{T}\right) \nabla_{\tau} u\right)_{\tau}\right)\right) \\
& +\partial_{n} f h_{2, n} h_{1, n}+f\left(h_{2, n} \partial_{n} h_{1, n}-\nabla_{\tau} h_{2, n} \cdot \mathbf{h}_{\mathbf{1}_{\tau}}\right) .
\end{aligned}
$$

From (A.11), we obtain

$$
\overline{\Delta_{\tau} u}=\Delta_{\tau} \dot{u}+\nabla_{\tau} \operatorname{div}_{\tau}\left(\mathbf{h}_{\mathbf{2} \tau}\right) \cdot \nabla_{\tau} u+\nabla_{\tau}\left(H \mathbf{h}_{2, n}\right) \cdot \nabla_{\tau} u-\operatorname{div}_{\tau}\left(\left(\left(D \mathbf{h}_{2}+\left(D \mathbf{h}_{2}\right)^{T}\right) \nabla_{\tau} u\right)_{\tau}\right),
$$

and using the relation between the material and shape derivative, we get

$$
\overline{\Delta_{\tau} u}=\Delta_{\tau} u^{\prime}+\nabla\left(\Delta_{\tau} u\right) \cdot \mathbf{h}_{2} \text { and } \Delta_{\tau} \dot{u}=\Delta_{\tau} u^{\prime}+\Delta_{\tau}\left(\nabla u \cdot \mathbf{h}_{2}\right) .
$$

Injecting these relations in (2.12) and applying them for $\mathbf{h}_{\mathbf{2}}$, we get

$$
\Delta_{\tau}\left(\nabla_{\tau} u \cdot \mathbf{h}_{\mathbf{2} \tau}\right)+\nabla_{\tau} \operatorname{div}_{\tau}\left(\mathbf{h}_{\mathbf{2} \tau}\right) \cdot \nabla_{\tau} u=\nabla_{\tau} \Delta_{\tau} u \cdot \mathbf{h}_{\mathbf{2} \tau}+\operatorname{div}_{\tau}\left(\left(\left(D \mathbf{h}_{2}+\left(D \mathbf{h}_{2}\right)^{T}\right) \nabla_{\tau} u\right)_{\tau}\right) .
$$

Thanks to this last fact, expression (2.11) gives:

$$
\begin{aligned}
\partial_{\mathbf{n}}\left(u_{1}^{\prime}\right)_{2}^{\prime}= & \operatorname{div}_{\tau}\left(h_{1, n} \nabla_{\tau} u_{2}^{\prime}+h_{2, n} \nabla_{\tau} u_{1}^{\prime}+\left(h_{2, n} \partial_{\mathbf{n}} h_{1, n}-\nabla_{\tau} h_{2, n} \cdot \mathbf{h}_{\mathbf{1}_{\tau}}\right) \nabla_{\tau} u\right) \\
& +\operatorname{div}_{\tau}\left(h_{1, n} h_{2, n}(H I-2 D \mathbf{n}) . \nabla_{\tau} u\right)+\partial_{n} f h_{2, n} h_{1, n}+f\left(h_{2, n} \partial_{n} h_{1, n}-\nabla_{\tau} h_{2, n} \cdot \mathbf{h}_{\mathbf{1}_{\tau}}\right) .
\end{aligned}
$$

To conclude, we use the following formula

$$
\partial_{\mathbf{n}} u_{1,2}^{\prime \prime}=\partial_{\mathbf{n}}\left(u_{1}^{\prime}\right)_{2}^{\prime}-\partial_{\mathbf{n}} u_{D \mathbf{h}_{1} \mathbf{h}_{2}}^{\prime}
$$

where

$$
\begin{aligned}
\partial_{\mathbf{n}} u_{D \mathbf{h}_{1} \mathbf{h}_{2}}^{\prime}= & \operatorname{div}_{\tau}\left(\left(h_{2, n} \mathbf{n} \cdot \nabla h_{1, n}+\nabla_{\tau} h_{1, n} \cdot \mathbf{h}_{\mathbf{2} \tau}-\mathbf{h}_{\mathbf{1} \tau} \cdot D \mathbf{n} \mathbf{h}_{\mathbf{2} \tau}\right) \nabla_{\tau} u\right) \\
& +f\left(h_{2, n} \partial_{n} h_{1, n}+\nabla_{\tau} h_{1, n} \cdot \mathbf{h}_{\mathbf{2} \tau}-\mathbf{h}_{\mathbf{1}_{\tau}} \cdot D \mathbf{n h}_{\mathbf{2} \tau}\right) .
\end{aligned}
$$

Finally, we obtain:

$$
\begin{aligned}
\partial_{\mathbf{n}} u_{1,2}^{\prime \prime}= & \operatorname{div}_{\tau}\left(h_{2, n} \nabla_{\tau} u_{1}^{\prime}+h_{1, n} \nabla_{\tau} u_{2}^{\prime}\right)-\operatorname{div}_{\tau}\left(\left(\mathbf{h}_{\mathbf{1}_{\tau}} \cdot \nabla_{\tau} h_{2, n}+\nabla_{\tau} h_{1, n} \cdot \mathbf{h}_{\mathbf{2} \tau}\right) \nabla_{\tau} u\right) \\
& -\operatorname{div}_{\tau}\left(h_{2, n} h_{1, n}(2 D \mathbf{n}-H I) \nabla_{\tau} u\right)+\operatorname{div}_{\tau}\left(\left(\mathbf{h}_{\mathbf{1}} \cdot D \mathbf{n} \mathbf{h}_{\mathbf{2}_{\tau}}\right) \nabla_{\tau} u\right) \\
& +\partial_{n} f h_{2, n} h_{1, n}-f\left(\nabla_{\tau} h_{2, n} \cdot \mathbf{h}_{\mathbf{1}_{\tau}}+\nabla_{\tau} h_{1, n} \cdot \mathbf{h}_{\mathbf{2}_{\tau}}-\mathbf{h}_{\left.\mathbf{2}_{\tau} \cdot D \mathbf{n h}_{\mathbf{1}_{\tau}}\right) .}\right.
\end{aligned}
$$

Proof of Corollary 1.3. It is completely similar to the proof of Theorem 1.1 without the volume right hand side $f$. Since $\Gamma_{o}$ remains fixed, no derivative of $u$ appears in that boundary condition. 


\subsection{Derivatives of the criterion. Proof of Theorem 1.4}

Proof of Proposition 1.2. We differentiate the definition of the criterion on the fixed domain $\Gamma_{o}$ and get

$$
D J_{L S}(\Omega ; \mathbf{h})=\int_{\Gamma_{o}} u^{\prime}\left(u-u_{0}\right)
$$

We introduce the adjoint state $p$ defined in (1.6) to write

$$
D J_{L S}(\Omega ; \mathbf{h})=\int_{\Gamma_{o}} u^{\prime} \partial_{n} p=\int_{\partial \Omega} u^{\prime} \partial_{n} p
$$

Since both $u^{\prime}$ and $p$ are harmonic, a double integration by parts makes possible to permute the normal derivation and leads to

$$
D J_{L S}(\Omega ; \mathbf{h})=\int_{\partial \Omega} \partial_{n} u^{\prime} p
$$

We insert the boundary conditions of (1.3) and use integration by part formula (A.6) to compute the gradient given in (1.5).

Proof of Theorem 1.4. To compute the second derivative of $J_{L S}$, we follow the classical scheme for computing the derivative for a boundary integral of a function $\psi$. The integral on $\Gamma_{m}^{t}$ can be brought back from the moving boundary $\Gamma_{m}^{t}$ to $\Gamma_{m}$ thanks to the change of variables

$$
\frac{1}{t}\left(I\left(\Gamma_{m}^{t}, h\right)-I\left(\Gamma_{m}\right)\right)=\frac{1}{t} \int_{\Gamma_{m}} \psi \circ T_{t} \omega(t)-\psi
$$

where $\omega(t)=\left|\operatorname{det}\left(D T_{t}\right)\right|\left\|\left(D T_{t}^{-1}\right)^{*} \mathbf{n}\right\|$. The useful formula $\omega^{\prime}(0)=\operatorname{div}_{\tau}(\mathbf{h})$ is stated in $[12,16]$.

In the case of $J_{L S}$, we apply this approach to $\psi=f p h_{n}-\nabla_{\tau} u . \nabla_{\tau} p h_{n}$ to get

$$
\frac{\mathrm{d}}{\mathrm{d} t}\left(D J_{L S}(\Omega ; \mathbf{h})\right)_{\mid t=0}=\frac{\mathrm{d}}{\mathrm{d} t}\left(\int_{\Gamma_{m}}\left[B(t) \nabla u^{t} \cdot \nabla p^{t}+f p\right] \mathbf{h} \circ T_{t} \cdot \mathbf{n}^{t}\right)_{\mid t=0}
$$

where we set $u^{t}=u\left(\Gamma_{m}^{t}\right) \circ T_{t}, p^{t}=p\left(\Gamma_{m}^{t}\right) \circ T_{t}, \mathbf{n}^{t}=\mathbf{n}\left(\Gamma_{m}^{t}\right) \circ T_{t}$ and $B(t)=\omega(t) D T_{t}^{-1}\left(D T_{t}^{-1}\right)^{*}$. Taking into account $\dot{\mathbf{n}}=-\left(D \mathbf{h}^{*}\right)_{\tau}$ and

$$
B^{\prime}(0)=\operatorname{div}_{\tau}(\mathbf{h}) I-D \mathbf{h}+(D \mathbf{h})^{*},
$$

we split the derivative of the shape derivative into three terms

$$
\frac{\mathrm{d}}{\mathrm{d} t}\left(D J_{L S}(\Omega ; \mathbf{h})\right)_{\mid t=0}=A_{1}(\Omega ; \mathbf{h})+A_{2}(\Omega ; \mathbf{h})+A_{3}(\Omega ; \mathbf{h})
$$

where

$$
\begin{aligned}
& A_{1}(\Omega ; \mathbf{h})=-\int_{\Gamma_{m}}\left[\nabla_{\tau} \dot{u} \cdot \nabla_{\tau} p+\nabla_{\tau} \dot{p} \cdot \nabla_{\tau} u+B^{\prime}(0) \nabla p \cdot \nabla u\right] h_{n} \\
& A_{2}(\Omega ; \mathbf{h})=\int_{\Gamma_{m}}(f p-\nabla u . \nabla p)\left(D \mathbf{h h} . \mathbf{n}-\mathbf{h} .\left(D h^{*} \mathbf{n}\right)_{\tau}\right) \\
& A_{3}(\Omega ; \mathbf{h})=\int_{\Gamma_{m}}\left[f p \operatorname{div}_{\tau}(\mathbf{h})+f \dot{p}+p \mathbf{h} . \nabla f\right] h_{n}=\int_{\Gamma_{m}}\left[f p \operatorname{div}_{\tau}(\mathbf{h})+f p^{\prime}+\mathbf{h} . \nabla(p f)\right] h_{n} .
\end{aligned}
$$


At the optimum, we know that $A_{2}\left(\Omega^{*} ; \mathbf{h}\right)=0$. Hence

$$
\begin{aligned}
D^{2} J_{L S}\left(\Omega^{*} ; \mathbf{h}, \mathbf{h}\right) & =\frac{\mathrm{d}}{\mathrm{d} t} D J_{L S}(\Omega ; \mathbf{h})_{\mid t=0}=A_{1}\left(\Omega^{*}, \mathbf{h}\right)+A_{3}\left(\Omega^{*}, \mathbf{h}\right), \\
& =-\int_{\Gamma_{m}}\left(\nabla_{\tau} \dot{u} \cdot \nabla_{\tau} p+\nabla_{\tau} \dot{p} \cdot \nabla_{\tau} u+B^{\prime}(0) \nabla p \cdot \nabla u\right) h_{n}+\left(f p \operatorname{div}_{\tau}(\mathbf{h})+f \dot{p}+p \mathbf{h} . \nabla f\right) h_{n} .
\end{aligned}
$$

Let us show how to simplify the expression. First of all, we write

$$
\nabla_{\tau} \dot{u} \cdot \nabla_{\tau} p=\left(\nabla u^{\prime}+D^{2} u \mathbf{h}+(D \mathbf{h})^{*} \nabla u\right) \cdot \nabla_{\tau} p
$$

and we then gather all the terms so that

$$
\nabla_{\tau} \dot{u} \cdot \nabla_{\tau} p+\nabla_{\tau} \dot{p} \cdot \nabla_{\tau} u=\nabla u^{\prime} \cdot \nabla_{\tau} p+\nabla u^{\prime} \cdot \nabla_{\tau} p+\mathbf{h} \cdot \nabla\left(\nabla_{\tau} u \cdot \nabla_{\tau} p\right)+\left(D \mathbf{h}+(D \mathbf{h})^{*}\right) \nabla p \cdot \nabla u,
$$

and then that

$$
A_{1}(\Omega ; h)=-\int_{\Gamma_{m}}\left[\nabla u^{\prime} \cdot \nabla_{\tau} p-\nabla u^{\prime} \cdot \nabla_{\tau} p-\mathbf{h} . \nabla\left(\nabla_{\tau} u . \nabla_{\tau} p\right)-\operatorname{div}_{\tau}(\mathbf{h}) \nabla_{\tau} u . \nabla_{\tau} p\right] h_{n} .
$$

We then obtain (1.8) by collecting the terms $A_{1}$ and $A_{3}$. We use Euler's equation to remark that

$$
\text { h. } \nabla\left(f p-\nabla_{\tau} u . \nabla_{\tau} p\right)=h_{n} \partial_{n}\left(f p-\nabla_{\tau} u . \nabla_{\tau} p\right) .
$$

\section{AnALysis OF STABILity}

\subsection{Shape hessian at a critical shape}

When $\Omega_{c}$ is a critical shape, the shape hessian takes the expression given by (1.8) that we recall here for convenience

$$
D^{2} J_{L S}\left(\Omega_{c} ; \mathbf{h}, \mathbf{h}\right)=\int_{\Gamma_{m}}\left(f p^{\prime}-\nabla u^{\prime} \cdot \nabla_{\tau} p-\nabla u \cdot \nabla_{\tau} p^{\prime}\right) h_{n}+\partial_{n}\left(f p-\nabla_{\tau} u . \nabla_{\tau} p\right) h_{n}^{2}
$$

This expression is difficult to deal with since the adjoint state is not well known at an arbitrary critical shape. Let us consider the particular case $\Omega_{c}=\Omega^{*}$ where the adjoint is simple.

Proof of Claim 1.5. Since at $\Omega^{*}, u=u_{0}$ by definition, the adjoint state $p$ cancels identically in $\Omega^{*}$ and from Corollary 1.3 so does its derivative $p^{\prime}$. Inserting these facts in (1.8), we get the claim.

\subsection{On the ersatz material approximation}

Our proof of Theorem 1.8 is inspired by Schwarz's method in domain decomposition. To that end, we introduce the operators

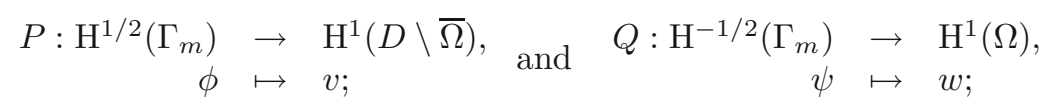

where $v$ and $w$ solves the boundary value problems

$$
\left\{\begin{array} { r l } 
{ \Delta v } & { = 0 \text { in } D \backslash \overline { \Omega } , } \\
{ v } & { = 0 \text { on } \Gamma _ { d } , } \\
{ \partial _ { n } v } & { = 0 \text { on } \Gamma _ { c } \cup \partial D , } \\
{ v } & { = \phi \text { on } \Gamma _ { m } , }
\end{array} \quad \text { and } \quad \left\{\begin{array}{rl}
\Delta w & =0 \text { in } \Omega, \\
w & =0 \text { on } \Gamma_{d}, \\
\partial_{n} w & =0 \text { on } \Gamma_{c}, \\
\partial_{n} w & =\psi \text { on } \Gamma_{m} .
\end{array}\right.\right.
$$


The classic theory of elliptic PDE learns us that both $P$ and $Q$ are bounded. Let $T_{\Gamma_{m}}$ is the usual trace operator from $\mathrm{H}^{1}(\Omega)$ into $\mathrm{H}^{1 / 2}\left(\Gamma_{m}\right)$. We then define the operators

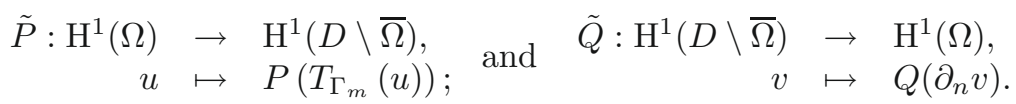

Note that $\tilde{Q}$ is not defined on the whole $\mathrm{H}^{1}(D \backslash \bar{\Omega})$ since the normal derivative $\partial_{n} v$ should have a sense. We will only apply the operator $\tilde{Q}$ to harmonic functions. In that case, the normal derivative is defined in the weak sense by duality. Moreover, if $v$ is harmonic in $D \backslash \bar{\Omega}$, then there is a constant $C(D \backslash \bar{\Omega})$ such that $\left\|\partial_{n} v\right\|_{\mathrm{H}^{-1 / 2}(\partial \Omega)} \leq C(D \backslash \bar{\Omega})\|v\|_{\mathrm{H}^{1}(D \backslash \bar{\Omega})}$ and then

$$
\|\tilde{Q}(v)\|_{\mathrm{H}^{1}(\Omega)} \leq C(D \backslash \bar{\Omega})\|Q\|\|v\|_{\mathrm{H}^{1}(D \backslash \bar{\Omega})} .
$$

Proof of Theorem 1.8. In fact, we will prove that $u_{\varepsilon}$ writes as a power series in the parameter $\varepsilon$. The idea is to split $u_{\varepsilon}$ into its values $U_{\varepsilon}$ on $\Omega$ and its values $V_{\varepsilon}$ on $D \backslash \bar{\Omega}$ and then to use a double ansatz as follows

$$
U_{\varepsilon}=\sum_{i=0}^{\infty} \varepsilon^{i} U_{\varepsilon, i} \text { and } V_{\varepsilon}=\sum_{i=0}^{\infty} \varepsilon^{i} V_{\varepsilon, i}
$$

The error estimate (1.13) is then easily deduced from the convergence of the series defined in (3.3). Let us prove that these series really converge.

We first determine the coefficients. Plugging these ansätze in the jump relations through the interface $\Gamma_{m}$ for the solution of the boundary value problem (1.10)

$$
\left[u_{\varepsilon}\right]=0 \text { and }\left[\sigma_{\varepsilon} \partial_{n} u_{\varepsilon}\right]=0,
$$

we get

$$
\begin{gathered}
U_{\varepsilon}=V_{\varepsilon} \Rightarrow \sum_{i=0}^{\infty} \varepsilon^{i} U_{\varepsilon, i}=\sum_{i=0}^{\infty} \varepsilon^{i} V_{\varepsilon, i} \\
\partial_{n} U_{\varepsilon}=\varepsilon \partial_{n} V_{\varepsilon} \Rightarrow \sum_{i=0}^{\infty} \varepsilon^{i} \partial_{n} U_{\varepsilon, i}=\sum_{i=1}^{\infty} \varepsilon^{i} \partial_{n} V_{\varepsilon, i-1} .
\end{gathered}
$$

By a term by term identification, we obtain $\partial_{n} U_{\varepsilon, 0}=0$ and the relationships

$$
\begin{aligned}
U_{\varepsilon, i} & =V_{\varepsilon, i}, \\
\partial_{n} V_{\varepsilon, i} & =\partial_{n} U_{\varepsilon, i+1}
\end{aligned}
$$

hold on $\Gamma_{m}$ for all $i \geq 1$.

As first consequence, $U_{\varepsilon, 0}$ solves the boundary value problem (1.1): it then holds $U_{\varepsilon, 0}=u$. The term of order zero of $V_{\varepsilon}$ is then completely characterized thanks to (3.4) as solution of the boundary value problem (3.1) with $\phi=T_{\Gamma_{m}} U_{\varepsilon, 0}$ where $T_{\Gamma_{m}}$ is the trace operator from $\mathrm{H}^{1}(\Omega)$ into $\mathrm{H}^{1 / 2}\left(\Gamma_{m}\right)$. We conclude that $V_{\varepsilon, 0}=\tilde{P}\left(U_{\varepsilon, 0}\right)$.

In fact, we obtain an iterative construction of the coefficients $V_{\varepsilon, i}$ and $U_{\varepsilon, i}$ in a Schwarz method's way. For all $i \geq 0,(3.4)$ implies that $V_{\varepsilon, i}=\tilde{P}\left(U_{\varepsilon, i}\right)$ and (3.5) that $U_{\varepsilon, i+1}=\tilde{Q}\left(V_{\varepsilon, i}\right)$. The coefficients in (3.3) are then completely defined by

$$
\left\{\begin{array} { l } 
{ U _ { \varepsilon , 0 } = u , } \\
{ U _ { \varepsilon , i + 1 } = \tilde { Q } \tilde { P } ( U _ { \varepsilon , i } ) \text { for } i \geq 0 ; }
\end{array} \text { and } \left\{\begin{array}{l}
V_{\varepsilon, 0}=\tilde{P}(u), \\
V_{\varepsilon, i+1}=\tilde{P} \tilde{Q}\left(V_{\varepsilon, i}\right) \text { for } i \geq 0 .
\end{array}\right.\right.
$$


Since the operator $\tilde{P}$ is bounded and thanks to (3.2), the expansions converge in the spaces $\mathrm{H}^{1}(\Omega)$ and $\mathrm{H}^{1}(D \backslash \bar{\Omega})$ for $\varepsilon$ small enough, for example such that

$$
\varepsilon<\frac{1}{C(D \backslash \bar{\Omega})\|\tilde{P}\|\|Q\|}
$$

\section{A. Appendix: Elements of shape calculus}

For the reader's convenience, we recall some basic facts from shape optimization without proof. We refer to $[12,16]$ for references and full proofs. Let $\mathbf{h}$ be a deformation field in $\mathrm{C}^{2}\left(\Omega, \mathbb{R}^{d}\right)$ with $\|\mathbf{h}\|_{\mathrm{C}^{2}}<1$. We set $T_{t}(\mathbf{h},)=.I d+t \mathbf{h}$ and denote by $\Omega_{t}$ the transported domain $\Omega_{t}=T_{t}(\Omega)$. It is well known that, for sufficiently small values of $t, T_{t}(\mathbf{h},$.$) is a diffeomorphism in \mathbb{R}^{d}$. To avoid heavy notations, we will misuse the notation $T_{t}$ instead of $T_{t}(\mathbf{h},$.$) .$

The key concepts we need are material and shape derivatives. For any vector field $\mathbf{h} \in \mathcal{H}$, we define the material derivative of the domain functional $y=y(\Omega)$ at $\Omega$ in an admissible direction $\mathbf{h}$ as the limit

$$
\dot{y}(\Omega ; \mathbf{h})=\lim _{t \rightarrow 0} \frac{y\left(\Omega_{t}\right) \circ T_{t}-y(\Omega)}{t} .
$$

Similarly, one can define the material derivative $\dot{y}(\partial \Omega ; \mathbf{h})$ for any domain functional $y=y(\partial \Omega)$ which depends on $\partial \Omega$. Another kind of derivative, called the shape derivative of $y(\Omega)$ in the direction $\mathbf{h}$, occurs. It is viewed as a first local variation. Its definition is given by the following

Definition A.1. The shape derivative $y^{\prime}=y^{\prime}(\Omega ; \mathbf{h})$ of a functional $y(\Omega)$ at $\Omega$ in the direction of a vector field $\mathbf{h}$ is given by

$$
y^{\prime}=\dot{y}-\mathbf{h} . \nabla y .
$$

We will need in the sequel to manipulate the tangential differential operators on a manifold. For the reader's convenience, we recall from $[12,16]$ some definitions and also some useful rules of calculus.

Definition A.2. The tangential divergence of a vector field $\mathbf{h} \in \mathrm{C}^{1}\left(\mathbb{R}^{d}, \mathbb{R}^{d}\right)$ is given by

$$
\operatorname{div}_{\tau}(\mathbf{h})=\operatorname{div}(\mathbf{h})-\text { Dh.n.n, }
$$

where the notation $D \mathbf{h}$ denotes the Jacobian matrix of $\mathbf{h}$. When the vector $\mathbf{h} \in \mathrm{C}^{1}\left(\partial \Omega, \mathbb{R}^{d}\right)$ is defined on $\partial \Omega$, then the following notation is used to define the tangential divergence

$$
\operatorname{div}_{\tau}(\mathbf{h})=\operatorname{div}(\tilde{\mathbf{h}})-(D \tilde{\mathbf{h}} \cdot \mathbf{n}) \cdot \mathbf{n},
$$

where $\tilde{\mathbf{h}}$ stands for an arbitrary $\mathrm{C}^{1}$ extension of $\mathbf{h}$ on an open neighbourhood of $\partial \Omega$.

We introduce the notion of tangential gradient $\nabla_{\tau}$ of a smooth scalar function $f$ in $\mathrm{C}^{1}\left(\partial \Omega, \mathbb{R}^{d}\right)$.

Definition A.3. Let an element $f \in \mathrm{C}^{1}\left(\partial \Omega, \mathbb{R}^{d}\right)$ be given and let $\tilde{f}$ be an extension of $f$ in the sense that $\tilde{f} \in \mathrm{C}^{1}(U)$ and $\left.\tilde{f}\right|_{\partial \Omega}=f$ and where $U$ is an open neighbourhood of $\partial \Omega$. Then the following notation is used to defined the tangential gradient

$$
\nabla_{\tau} f=\left.\nabla \tilde{f}\right|_{\partial \Omega}-\nabla \tilde{f} . \mathbf{n} \mathbf{n} \text { on } \partial \Omega .
$$

The details for the existence of such an extension can be found in [12]. Let us remark that these definitions do not depend on the choice of the extension. Furthermore, one can show the important relation

$$
\int_{\partial \Omega} \nabla_{\tau} f \cdot \mathbf{F}=-\int_{\partial \Omega} f \operatorname{div}_{\tau}(\mathbf{F})
$$


for all elements $f \in \mathrm{C}^{1}(\partial \Omega)$ and all vector fields $F \in \mathrm{C}^{1}\left(\partial \Omega, \mathbb{R}^{d}\right)$ satisfying $F_{n}=\langle F, n\rangle=0$. In general, the condition above $F_{n}=0$ is not always satisfied. The extension of this integration by parts formula to fields with a normal vector component involves curvature. First, we point out that the curvature is connected to the normal vector via the tangential divergence operator. Recall that the mean curvature of $\partial \Omega$ is defined as $H=\operatorname{div}_{\tau}(\mathbf{n})$. Making use of the form of $\operatorname{div}_{\tau}(\mathbf{n})$ on the boundary, one shows straightforwardly the following statement.

Proposition A.4. Let $\Omega$ be an open subset of $\mathbb{R}^{3}$ with a $\mathrm{C}^{2}$ boundary. For any unitary extension $\mathcal{N}$ of $n$ on a neighbourhood of $\partial \Omega$, one has

$$
\operatorname{div}(\mathcal{N})=H \text { on } \partial \Omega
$$

Assume that the manifold $\partial \Omega$ has no boundary. If $\mathbf{F} \in \mathrm{H}^{2}(\partial \Omega)^{3}$ and $f \in \mathrm{H}^{2}(\partial \Omega)$, then we have

$$
\int_{\partial \Omega} \nabla f . \mathbf{F}+f \operatorname{div}_{\tau}(\mathbf{F})=\int_{\partial \Omega}(\nabla f . \mathbf{n}+H f) \mathbf{F} . \mathbf{n} .
$$

We assume now that the domain $\Omega$ has a $\mathrm{C}^{3}$ boundary. The simplest second-order derivative is the Laplace Beltrami operator; it is defined as follows thanks to the following usual chain rule.

Definition A.5. Let $f \in H^{2}(\partial \Omega)$. The Laplace-Beltrami $\Delta_{\tau}$ of $f$ is defined as follows

$$
\Delta_{\tau} f=\operatorname{div}_{\tau}\left(\nabla_{\tau} f\right) .
$$

There is a relation connecting the Laplace operator and the Laplace-Beltrami operator. Let us denote by $\partial_{n n}^{2} f=\left(D^{2} f . \mathbf{n}\right) . \mathbf{n}$ where $D^{2} f$ stands for the hessian of $f$.

Proposition A.6. Let $\Omega$ be a domain with a boundary $\partial \Omega$ of class $\mathrm{C}^{3}$. For all functions $f \in H^{3}(\Omega)$, it holds

$$
\Delta f=\Delta_{\tau} f+H \partial_{\mathbf{n}} f+\partial_{n n}^{2} f, \text { on } \partial \Omega .
$$

We need to compute shape and material derivative of special vector fields: the outer unit normal vector $\mathbf{n}$, the tangential gradient and the Laplace-Beltrami operator applied to a function. While the derivative of the normal vector is obtained by a straightforward calculus, we have to transport from $\partial \Omega_{t}$ to $\partial \Omega$ the Laplace-Beltrami operator and the tangential gradient in order to compute the other derivatives. We recall here facts proved in $[2]$.

We denote by $\mathbf{n}$ the gradient of the signed distance to $\partial \Omega$. This is an unitary extension of the unitary normal vector $\mathbf{n}$ at $\partial \Omega$ which is smooth in the vicinity of $\partial \Omega$. This extension furnishes a symmetric Jacobian $D \mathbf{n}$ that satisfies $D \mathbf{n} \mathbf{n}=0$ on $\partial \Omega$. The direction $\mathbf{h}$ will be supposed to be in $\mathrm{C}^{2}\left(\mathbb{R}^{d}, \mathbb{R}^{d}\right)$ or in $\mathrm{C}^{2}\left(\partial \Omega, \mathbb{R}^{d}\right)$. We recall:

\section{Proposition A.7.}

- The material derivative $\dot{\mathbf{n}}$ of the normal vector $\mathbf{n}$ at $\Omega$ in the direction of a vector field $\mathbf{h} \in \mathrm{C}^{1}\left(\mathbb{R}^{d}, \mathbb{R}^{d}\right)$ is given by

where $\mathbf{h}_{\tau}=\mathbf{h}-\mathbf{h} . \mathbf{n} \mathbf{n}$.

$$
\dot{\mathbf{n}}=-\nabla_{\tau}(\mathbf{h} . \mathbf{n})+D \mathbf{n} \mathbf{h}_{\tau},
$$

- The shape boundary $\mathbf{n}^{\prime}$ in the direction of $\mathbf{h}$ is given by

$$
\mathbf{n}^{\prime}=-\nabla_{\tau}(\mathbf{h} . \mathbf{n}) .
$$

- For all functions $f \in \mathrm{C}^{2}\left(\mathbb{R}^{3}\right)$ and directions $\mathbf{h} \in \mathrm{C}^{2}\left(\partial \Omega, \mathbb{R}^{3}\right)$, one has

$$
\dot{\overline{\nabla_{\tau} f}}=\nabla \dot{f}+\left(D^{2} f \mathbf{h}\right)_{\tau}-\nabla f . \mathbf{n} \dot{n}-\nabla f . \dot{\mathbf{n}} \mathbf{n} .
$$


- Let $f \in \mathcal{D}\left(\mathbb{R}^{d}\right)$. The material derivative of $\Delta_{\tau} f$ in the direction $\mathbf{h}$ is given by

$$
\overline{\overline{\Delta_{\tau} f}}=\Delta_{\tau} \dot{f}+\nabla_{\tau} f . \nabla_{\tau}\left[\operatorname{div}_{\tau}\left(\mathbf{h}_{\tau}\right)\right]+\nabla_{\tau}\left(H \mathbf{h}_{n}\right) . \nabla_{\tau} f-\operatorname{div}_{\tau}\left(\left(\left(D \mathbf{h}+(D \mathbf{h})^{T}\right) \nabla_{\tau} f\right)_{\tau}\right) .
$$

In the sequel, we will use some technical formulae given in [2]. Given a smooth vector field $\mathbf{h}$, we denote

$$
A_{\mathbf{h}}=D \mathbf{h}+D \mathbf{h}^{T}-\operatorname{div}(\mathbf{h}) I .
$$

Given two smooth vector fields $\mathbf{h}_{1}$ and $\mathbf{h}_{2}$, we set

$$
\mathcal{A}=D \mathbf{h}_{2} A_{\mathbf{h}_{1}}+A_{\mathbf{h}_{1}} D \mathbf{h}_{2}{ }^{T}-A_{\mathbf{h}_{1}} \operatorname{div}\left(\mathbf{h}_{2}\right)-\left(A_{\mathbf{h}_{1}}\right)^{\prime}\left(\mathbf{h}_{2}\right),
$$

and

$$
b=\left(\mathbf{h}_{2} . \nabla u\right) A_{\mathbf{h}_{1}} \nabla v+\left(\mathbf{h}_{2} . \nabla v\right) A_{\mathbf{h}_{1}} \nabla u-\left(\left(A_{\mathbf{h}_{1}} \nabla u\right) . \nabla v\right) \mathbf{h}_{2} .
$$

Here, the notation $\left(A_{\mathbf{h}_{1}}\right)^{\prime}\left(\mathbf{h}_{2}\right)$ stands for the matrix defined by its elements

$$
\left(\left(A_{\mathbf{h}_{1}}\right)^{\prime}\left(\mathbf{h}_{2}\right)\right)_{k, l}=\nabla\left(\left(\left(A_{\mathbf{h}_{1}}\right)^{\prime}\right)_{k, l}\right) \cdot \mathbf{h}_{2} .
$$

Proposition A.8. It holds:

$$
\begin{gathered}
\nabla u \cdot A_{\mathbf{h}} \nabla v=\nabla(\mathbf{h} . \nabla u) . \nabla v+\nabla(\mathbf{h} . \nabla v) \nabla u-\operatorname{div}((\nabla u . \nabla v) \mathbf{h}) . \\
\nabla u \cdot \mathcal{A} \nabla v=\operatorname{div}(b)-\left(\mathbf{h}_{2} \cdot \nabla u\right) \operatorname{div}\left(\left(A_{\mathbf{h}_{1}} \nabla v\right)\right)-\left(\mathbf{h}_{2} . \nabla v\right) \operatorname{div}\left(\left(A_{\mathbf{h}_{1}} \nabla u\right)\right) .
\end{gathered}
$$

For any function $u$ solution of (1.1) in $\Omega$ and for every test function $\phi \in \mathcal{D}(\Omega)$, we have

$$
\operatorname{div}\left(A_{\mathbf{h}_{1}} \nabla u\right)=\Delta\left(\mathbf{h}_{1} \cdot \nabla u\right)+\operatorname{div}\left(f \mathbf{h}_{1}\right) .
$$

\section{REFERENCES}

[1] L. Afraites, M. Dambrine, K. Eppler and K. Kateb, Detecting perfectly insulated obstacles by shape optimization techniques of order two. Discret. Contin. Dyn. Syst. - série B 8 (2007) 389-416.

[2] L. Afraites, M. Dambrine and D. Kateb, On second order shape optimization methods for electrical impedance tomography. SIAM J. Control Optim. 47 (2008) 1556-1590.

[3] G. Allaire and F. Jouve, A level-set method for vibration and multiple loads in structural optimization. Comput. Methods Appl. Mech. Engrg. 194 (2005) 3269-3290.

[4] G. Allaire, F. Jouve and A.-M. Toader, Structural optimization using sensitivity analysis and a level-set method. J. Comput. Phys. 194 (2004) 363-393.

[5] P. Bernardoni, Outils et méthode de conception de structures mécaniques à déformations et actionnements répartis. Ph.D. Thesis, Université Paris VI, France (2004).

[6] D. Bucur, Do optimal shapes exist? Milan J. Math. 75 (2007) 379-398.

[7] P. Cardaliaguet and O. Ley, Some flows in shape optimization. Arch. Ration. Mech. Anal. 183 (2007) 21-58.

[8] P. Cardaliaguet and O. Ley, On the energy of a flow arising in shape optimization. Interfaces Free Bound. 10 (2008) $221-241$.

[9] M. Dambrine, About the variations of the shape Hessian and sufficient conditions of stability for critical shapes. Revista Real Academia Ciencias-RACSAM 96 (2002) 95-121.

[10] M. Dambrine and M. Pierre, About stability of equilibrium shapes. ESAIM: M2AN 34 (2000) 811-834.

[11] F. de Gournay, Velocity extension for the level-set method and multiple eigenvalues in shape optimization. SIAM J. Control Optim. 45 (2006) 343-367.

[12] M. Delfour and J.P. Zolesio, Shapes and Geometries: Analysis, Differential Calculus, and Optimization. SIAM (2001).

[13] J. Descloux, Stability of the solutions of the bidimensional magnetic shaping problem in abscence of surface tension. Eur. J. Mech. B Fluid. 10 (1991) 513-526. 
[14] K. Eppler and H. Harbrecht, A regularized newton method in electrical impedance tomography using hessian information. Control Cybern. 34 (2005) 203-225.

[15] K. Eppler, H. Harbrecht and R. Schneider, On convergence in elliptic shape optimization. SIAM J. Control Optim. 46 (2007) 61-83.

[16] A. Henrot and M. Pierre, Variation et optimisation de formes, Mathématiques et Applications 48. Springer (2005).

[17] F. Hettlich and W. Rundell, A second degree method for nonlinear inverse problems. SIAM J. Numer. Anal. 37 (1999) 587-620.

[18] V. Isakov, Inverse problems for partial differential equations, Applied Mathematical Sciences 127. Springer (2006).

[19] A. Kisch, The domain derivative and two applications in inverse scattering theory. Inverse Problems 9 (1993) 81-96.

[20] S. Osher and J.A. Sethian, Fronts propagating with curvature dependent speed: algorithms based on Hamilton-Jacobi formulations. J. Comput. Phys. 79 (1988) 12-49. 